\title{
Dresdner Dermatologische Demonstration 2009 - zugleich Tagung der Sächsischen Dermatologischen Gesellschaft am 7. März 2009
}

\section{Dresden Dermatology Demonstration 2009 - Meeting of the Saxonian Society of Dermatology, March 7, 2009}

Vorsitz:

Berichterstatter:

Histopathologie:

Klinische Photodokumentation:

Plenarvorträge:

\section{U. Wollina}

G. Hansel, A. Koch, U. Wollina

J. Schönlebe, G. Haroske

R. Herz

J. Wohlrab, Halle/Saale Topische Therapie - Stand, Neuigkeiten und Ausblicke

L. Dourmishev, Sofia/Bulgarien Syphilis - eine Übersicht zu seltenen und ungewöhnlichen klinischen Verläufen

E. Stockfleth, Berlin Aktinische Keratosen - kosmetischer Makel oder neoplastischer Marker?

\section{Bibliografie}

DOI $10.1055 / \mathrm{s}-0029-1214710$ Online-Publikation: 26.5.2009 Akt Dermatol 2009; 35: 403-410 @ Georg Thieme Verlag KG Stuttgart · New York ISSN 0340-2541

\section{Korrespondenzadresse} Prof. Dr. Uwe Wollina Klinik für Dermatologie und Allergologie am Krankenhaus Dresden-Friedrichstadt Friedrichstraße 41 01067 Dresden wollina-uw@khdf.de

\section{Tinea incognito}

$\nabla$

\section{J. Runge, I. Vennewald, G. Hansel}

Im November 2007 waren bei der 73-jährigen Patientin erstmals flechtenartige Hautveränderungen am linken distalen Unterschenkel aufgetreten. Seit März 2008 wurde eine zunehmende Progression mit einer tiefen Ulzeration prätibial am linken Unterschenkel beobachtet ( $\bullet$ Abb. 1). An Nebenerkrankungen waren ein arterieller Hypertonus, eine Varikose beidseits und eine Hypothyreose bekannt.

Hautbefund: Bei Aufnahme Anfang November 2008 zeigte sich am linken Unterschenkel ein girlandenförmiges, randbetontes Erythem, welches fast die gesamt Zirkumferenz einnahm. Zusätzlich bestand eine grobe Schuppung. Distal davon fand sich ein sichelförmiges, relativ tiefes, scharf begrenztes Ulkus mit Fibrinbelägen. Die Fußund Fingernägel waren dystroph, grau-gelblich verfärbt und teilweise abgebrochen.

Histologie: Wir führten diagnostische Exzisionen durch. Im Bereich des Papillarkörpers und oberen Koriums fand sich ein herdbetont außerordentlich schütteres lymphomonozytoides entzündliches Infiltrat in vorzugsweise perivaskulärer Lokalisation. Im Stratum corneum Nachweis zahlloser Pilzhyphen ( Abb. 2).

Laborbefunde: Pathologisch waren Kreatinin (109,6 $\mu \mathrm{mol} / \mathrm{l})$, GFR (46,3), Harnstoff (9,3 mmol/l) und Thrombozyten (408,0 Gpt/1).

Mykologie: Nagelspäne und Hautschuppen (๑ Abb. 3, $\odot$ Tab. 1).

Therapie und Verlauf: Lokaltherapie mit Ciclopiroxolamin (Batrafencreme ${ }^{\circledR}$ ), interne Therapie mit Itraconazol $200 \mathrm{mg} / \mathrm{d}$ (Sempera $\left.{ }^{\circledR}\right)$. Die Therapie wurde über 3 Monate kontinuierlich angewendet und gut toleriert. Bei einer ambulanten Vorstellung Mitte Januar 2009 stellte sich die Patientin mit einem komplett abgeheilten Hautbefund vor.

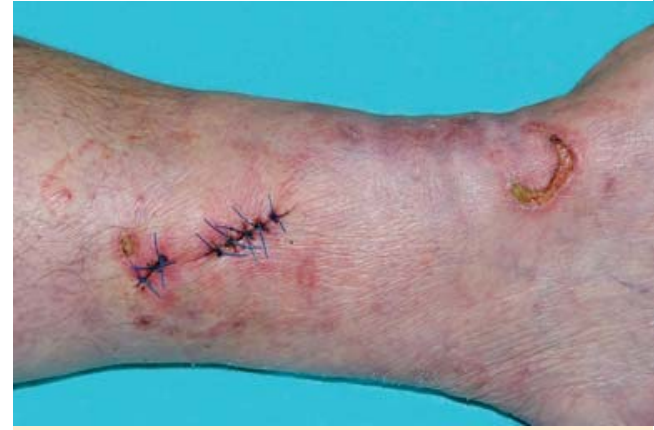

Abb. 1 Lokalbefund linker Unterschenkel medial.

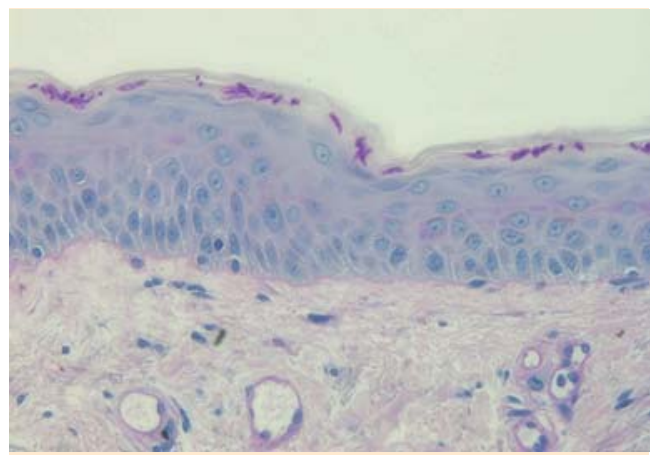

Abb. 2 Histologie mit Pilzelementen im Stratum corneum (PAS, $\times 40$ ).

Kommentar: Die Tinea incognito wurde 1968 von Ive et al. [1] erstmals beschrieben. Der klinischmorphologische Befund ist dabei durch die symptomatische Anwendung topischer Glukokortikoide verändert. Der Pilz sitzt in der Hornschicht und metabolisiert das Keratin, dadurch wird in der darunter liegenden Epidermis eine Ekzemreaktion ausgelöst. Die typischen Entzündungszeichen werden durch die antiinflammatorisch wirkenden Kortikoide unterdrückt, sodass es zu Fehldiagnosen kommen kann [2,3]. In den von Ive et al. beschriebenen Fällen wurden z.B. 


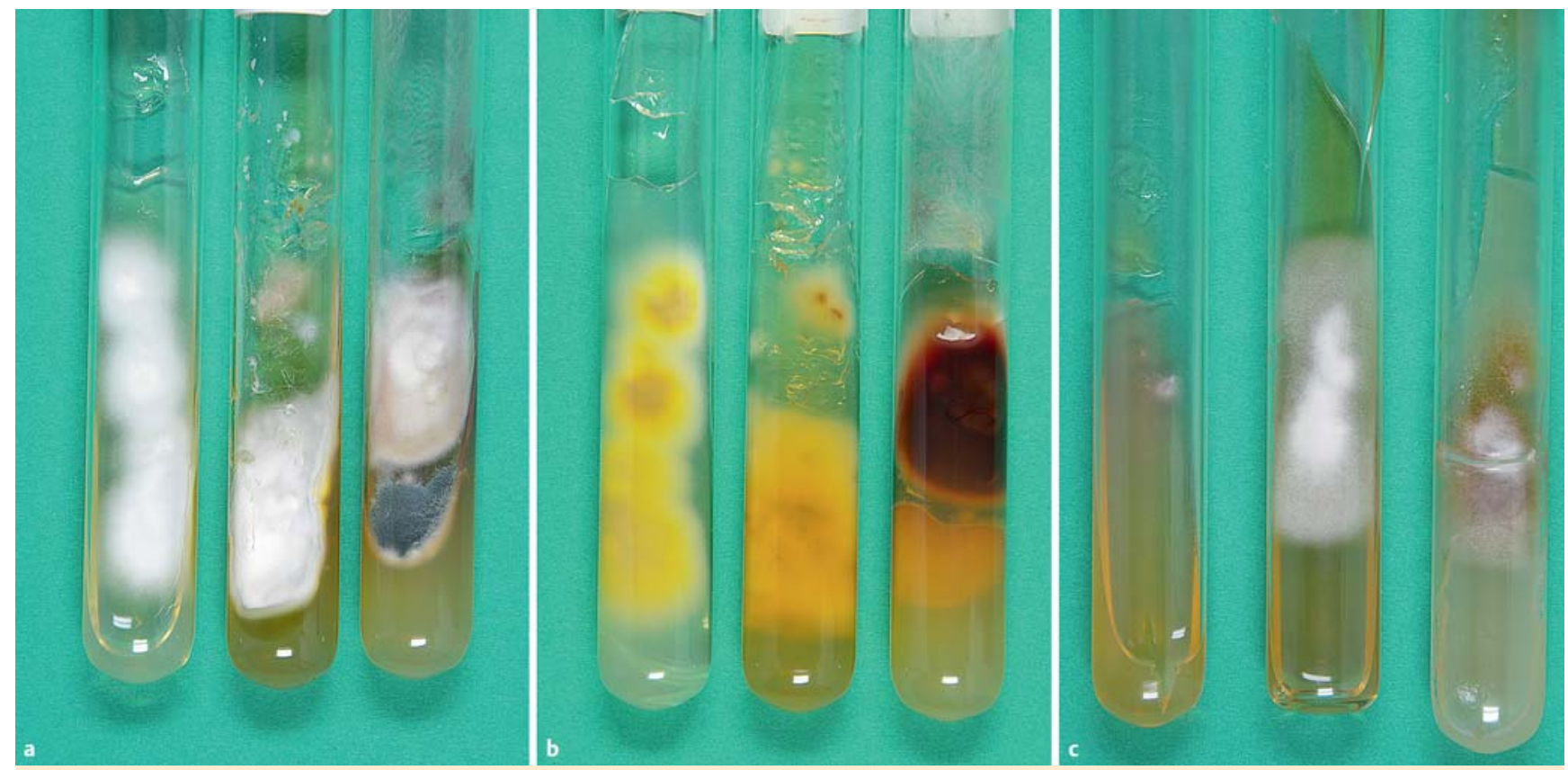

Abb. 3 In (a) sieht man das typische weiße, flauschige Wachstum von Trichophyton rubrum auf allen 3 Nährböden (Sabouraud-, Kimmig- und Mycoselagar), ganz rechts ist zusätzlich Aspergillus fumigatus zu sehen (blau-grün); (b) stellt die Rückseite von (a) dar. Dabei zeigt sich das imposante rot-braune, kreisrunde Wachstum der T. rubrum-Kolonie auf Sabouraud-Agar. Auf Mycozel-Agar (links) ist das kreisförmige, gelb-braune Wachstum von T. rubrum zu sehen. (c) zeigt die biochemische Differenzierung mit Kartoffel- (rechts), Malz- (Mitte) und Harnstoffagar (links). Auf dem Kartoffelagar zeigt sich eine typische braun-rötliche Verfärbung. Harnstoff blieb negativ. Nach 7 Tagen kann sich bei T. rubrum eine zartrosa Färbung zeigen, im Gegensatz zu anderen Trichophytonarten.

Tab. 1 Mykologischer Befund.

\begin{tabular}{|c|c|c|}
\hline Lokalisation & mikroskopisch & kulturell \\
\hline $\begin{array}{l}\text { 1. Finger li } \\
\text { Hand }\end{array}$ & $\begin{array}{l}\text { vereinzelt } \\
\text { Dermatophyten }\end{array}$ & $\begin{array}{l}\text { vereinzelt Candida } \\
\text { parapsilosis }\end{array}$ \\
\hline $\begin{array}{l}\text { 3. Finger li } \\
\text { Hand }\end{array}$ & $\begin{array}{l}\text { sehr viele } \\
\text { Dermatophyten }\end{array}$ & $\begin{array}{l}\text { viel Candida parapsilosis, } \\
\text { viel Candida lipolytica, } \\
\text { vereinzelt Rhodotorula rubra }\end{array}$ \\
\hline 4. Zehe li & $\begin{array}{l}\text { sehr viele } \\
\text { Dermatophyten }\end{array}$ & $\begin{array}{l}\text { vereinzelt Candida glabrata, } \\
\text { Trichophyton rubrum } \\
\text { und Cryptococcus }\end{array}$ \\
\hline li Großzehe & viel Dermatophyten & $\begin{array}{l}\text { Aspergillus (evtl. Anflug- } \\
\text { keim) }\end{array}$ \\
\hline li US dorsal & $\begin{array}{l}\text { sehr viele } \\
\text { Dermatophyten }\end{array}$ & Trichophyton rubrum \\
\hline li Handballen & keine Pilzelemente & Pilzkultur negativ \\
\hline
\end{tabular}

eine papulöse Rosazea oder eine Poikilodermie diagnostiziert.

Bei der Diagnostik steht der mykologische Nachweis im Nativpräparat und in der Kultur an erster Stelle.

Der Trend zur Zunahme der Tinea incognito könnte auf eine mangelhafte Diagnostik beim erstbehandelnden Arzt sowie den unkritischen Gebrauch in Therapie und Selbstmedikation der topischen Kortikoide zurückzuführen sein.

\section{Literatur}

1 Ive A, Marks R. Tinea incognito. Br Med J 1986; 3: 149-152

2 Wacker J, Durani BK, Hartschuh W. Bizarre annular lesion emerging as tinea incognito. Mycoses 2004; 47: 447-449

2 Hansel G, Vennewald I, Wollina U. Tinea incognito. G Ital Dermatol Venereol 2004; 139: 263

\section{Kerion Celsi bei Tinea capitis et corporis}

\section{S. Klimke, I. Vennewald, A. Koch}

Vor 5 Wochen bemerkte die Mutter des 8-jährigen Patienten erstmals Hautveränderungen auf der Kopfhaut, die sich später auf das Gesicht, die rechte Schulter und beide Arme ausbreiteten. Es bestand Juckreiz. Sie berichtete von einem Pilzbefall des Haustieres (Hase) in der Vergangenheit. Eine Behandlung war bereits erfolgt.

Eine Mikrosporie war zunächst ambulant vermutet worden. Der Patient erhielt systemisch Flukonazol (Diflucan ${ }^{\circledR}$ ) $50 \mathrm{mg} / \mathrm{d}$ ohne genügendes therapeutisches Ansprechen.

Allgemeinbefund:

Der Patient war in gutem Allgemein- und Ernährungszustand, kein Fieber, keine Lymphknotenschwellung.

Hautbefund: Es befanden sich ein großer erythemato-squamöser, stark verkrusteter Herd auf der rechtsseitigen Kopfhaut und weitere kleinere Herde am übrigen Kopf ( $\bullet$ Abb.4).

Außerdem bestanden ekzematöse Herde an Armen, Wange und der Schulter. Das Integument war insgesamt trocken.

\section{Mykologie:}

Mikroskopie: Schuppen Kopf: eindeutig Dermatophyten sehr viel, Haare - ektotricher Befall ( $\bullet$ Abb.5).

Pilzkultur: Trichophyton mentagrophytes variatio asteroides (৫ Abb. 6).

Therapie und Verlauf: Nach Aufnahme und diagnostischen Maßnahmen wurden die Verkrustungen am Kopf entfernt und die Haare gekürzt. Lokal kam Ciclopiroxolamin (Batrafencreme ${ }^{\circledR}$ ), systemisch Flokonazol (Diflucan ${ }^{\circledR}$ ) $50 \mathrm{mg} / \mathrm{d}$ zur Anwendung, darunter deutliche klinische Besserung. 


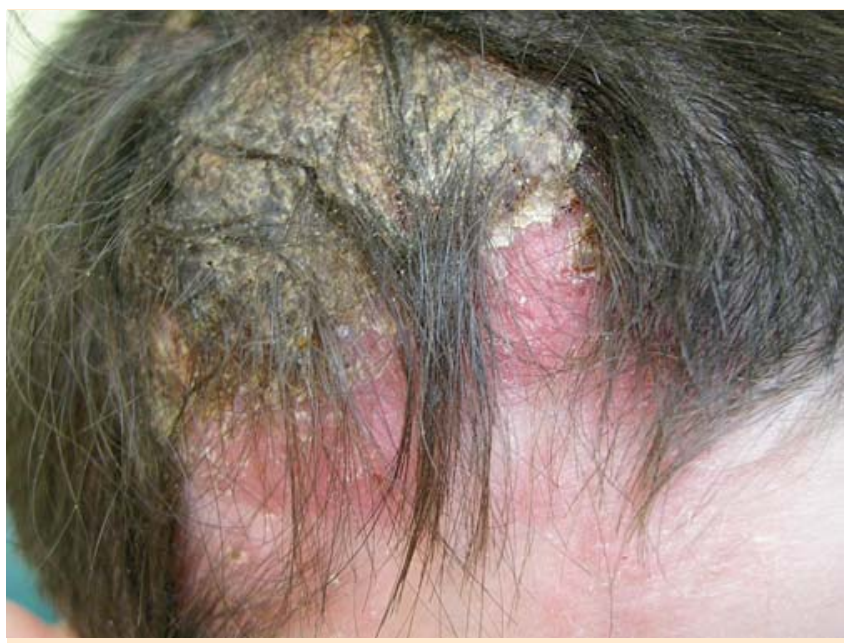

Abb. 4 Kerion Celsi.

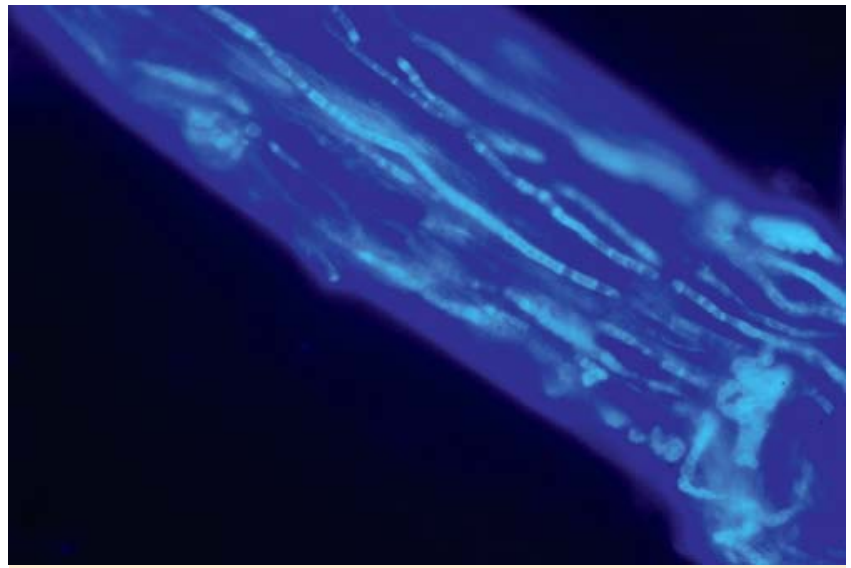

Abb. 5 Pilzkultur Trichophyton mentagrophytes var. asteroides, weiße und leicht gekörnte Kulturoberfläche.

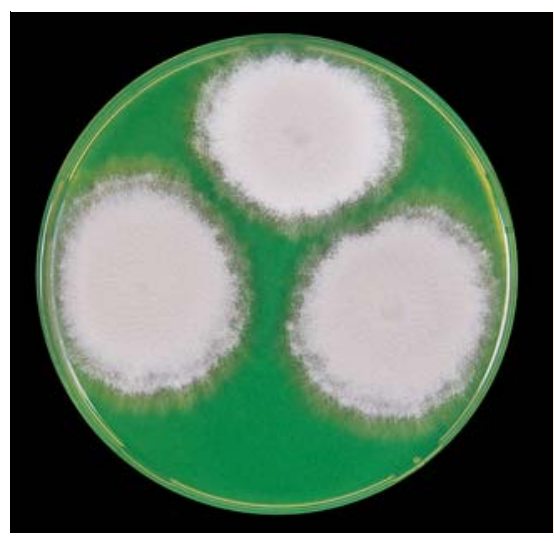

Abb. 6 Fluoreszenzmikroskopisches Bild des ektotrichen Haarbefalls.

Zur ambulanten Weiterbehandlung wurde die systemische Therapie mit Flukonazol $50 \mathrm{mg} / \mathrm{d}$ oder Griseofulvin $10 \mathrm{mg} / \mathrm{kg}$ und Tag empfohlen.

Kommentar: Die Tinea capitis et corporis ist eine hochkontagiöse Mykose des Kindesalters. Haupterreger in Europa sind zoophile Dermatophyten wie Microsporum canis und Trichophyton mentagrophytes. Die Krankheit tritt sowohl sporadisch als auch endemisch auf [2].

Die Tinea capitis microsporica zeigt runde, einzeln oder multipel z. T. konfluierende Herde, die Haare sind 2-3 mm über der Haut- oberfläche abgebrochen. Die Tinea capitis superficialis trichophytica imponiert durch unregelmäßig gestaltete erythematosquamöse Herde, die Haare brechen unmittelbar im Hautniveau. Im Nativpräparat der Haare lassen sich ektotricher (Microsporium canis, Trichophyton mentagrophytes) von endotrichem (Trichophyton tonsurans, T. violaceum, T. mentagrophytes) Pilzbefall unterscheiden.

Eine diagnostische Hilfe ist das Wood-Licht, bei gelblich-grüner Fluoreszenz ist eine Mikrosporum-Erkrankung wahrscheinlich. Die endgültige Diagnose wird durch die Pilzkultur gesichert [1]. Die Tinea capitis muss nach gegenwärtiger Auffassung stets systemisch und zusätzlich lokal behandelt werden [3]. Griseofulvin besitzt in Deutschland die Zulassung zur systemischen Therapie der kindlichen Tinea capitis. Aufgrund der guten Verträglichkeit und Verkürzung der Therapiezeit eignen sich jedoch auch andere Antimykotika wie Itraconazol, Terbinafin und Fluconazol zur Anwendung über 4-8 Monate im Rahmen eines individuellen Heilversuchs [1,3]. Beispielsweise ist Itraconazol dem Griseofulvin in Hinblick auf Sicherheit und Effizienz zumindest gleichwertig und hat den Vorteil einer kürzeren Behandlungsdauer [3]. Microsporum canis-Isolate zeigen eine sehr hohe und dem Griseofulvin deutlich überlegene Empfindlichkeit gegenüber Terbinafin [4].

Die Lokaltherapie mit Ciclopiroxolamin oder Terbinafin reduziert das Übertragungsrisiko. Zusammen mit der Entfernung der Haare und der regelmäßigen Haarwäsche mit antimykotisch wirksamen Shampoos verkürzt das die Behandlungsdauer [1].

\section{Literatur}

1 Seebacher C, Abeck D, Brasch J et al. Tinea capitis: ringworm of the scalp. Mycoses 2007; 50: 218-226

2 Ginter-Hanselmayer G, Weger W, Ilkit M, Smolle J. Epidemiology of tinea capitis in Europe: current state and changing pattern. Mycoses 2007; 50 (Suppl 2): 6-13

3 Korting $\mathrm{H}-\mathrm{C}$, Schöllmann C. Stellenwert von Itraconazol in der Behandlung von Pilzinfektionen der Haut, Nägel und Schleimhäute. J Dtsch Dermatol Ges 2009; 7: $11-20$

4 Wollina U, Vennewald I. Kreisrunder Haarausfall beim Kind: Tinea capitis durch Microsporum canis. Hautnah Dermatol 2004; 20 : $312-312$

\section{Pemphigus foliaceus}

$\nabla$

\section{G. Hansel, ]. Schönlebe}

Erstmals nahmen wir die 61-jäjhrige Patientin im September 2007 mit generalisiert erythematösen, teils schuppenden, juckenden, seit 2 Monaten bestehenden Hautveränderungen auf. Diese hätten sich von den Schultern und dem Dekolleté ausgehend langsam ausgebreitet und würden durch Sonneneinstrahlung verschlechtert. Anfänglich hätte sie kleine Bläschen beobachtet. Sie war bei mehreren Hautärzten gewesen, hatte schon Prednisolon und Teersalben erhalten. Eine trockene Haut bestünde seit vielen Jahren und würde regelmäßige Pflege brauchen.

Unter Lokalbehandlung mit kortikosteroidhaltigen Externa besserte sich der Hautbefund zögerlich. Wegen akuter Exazerbation einer COPD mussten Prednisolon und Cefuroxim ordiniert werden. Darunter bildete sich der Hautbefund zurück. Kurzzeitig lokalisiert auftretende Pusteln gaben Anlass zu einer diagnostischen Exzision, welche lediglich eine Ekzemreaktion ergab. Bei stummer Immunfluorezenz schien auch ein differenzialdiagnostisch berücksichtigter Lupus erythematodes ausgeschlossen. Mit 
raschen Rezidiven wurde die Patientin zweimal wieder eingewiesen, eine erneute histologische Untersuchung brachte keine neuen Gesichtspunkte. Die Hautveränderungen erfassten auch das Gesicht und waren teilweise massiv superinfiziert, führten insbesonder an der Brust zu stark entzündlichen Infiltraten.

An Begleiterkrankungen waren neben der COPD eine arterielle Hypertonie und eine absolute Arrythmie bei Vorhofflimmern bekannt. Im Verlaufe entwickelten sich eine latente Hyperthyreose und eine entzündlich bedingte Anämie. Die Wunden und Schleimhäute besiedelten mit methicillinrestentem Staphylococcus aureus.

Ende Januar 2008 wurde die Patientin zum vierten Male eingewiesen.

Allgemeinbefund: Bei stationärer Aufnahme befand sich die adipöse Patientin durch die ausgedehnte Hauterkrankung stets in reduziertem Allgemeinzustand.

Hautbefund: Bei der klinischen Untersuchung sahen wir am gesamten Integument entzündliche bräunlich-rötliche, von Krusten bedeckte, z. T. großflächig konfluierende Infiltrate unter Einbeziehung von Kopf- und Gesichtshaut ( $\bullet$ Abb. 7).

Palmae, Plantae und Schleimhaute waren ausgespart. Bläschen und Pusteln bestanden nicht, obwohl die Patientin wiederum berichtete, zu Beginn des Schubes Bläschen beobachtet zu haben. Das Nikolski-Phänomen war negativ. Mittels nochmaliger diagnostischer Exzision konnte die Diagnose nach 4 Monaten geklärt werden.

Histologie: Epidermis mäßiggradig, teils uncharakteristisch, teils angedeutet psoriasiform hyperplastisch mit erhaltenem Stratum granulosum und breitflächig aufgelagerter leukozytenreicher herdförmig bakteriell besiedelter Schuppenkruste, die auch eine kleinherdige Erosion abdeckt. Wechselnd dichtes überwiegend perivaskulär lokalisiertes entzündliches Infiltrat aus überwiegend lymphomonozytoiden Zellen untermischt von wenigen neutrophilen und eosinophilen Granulozyten. Kurzstreckige subcorneale Spaltbildung mit angedeuteter Akantholyse oberflächlicher Keratinozyten ( $\bullet$ Abb. 8).

Direkte Immunfluoreszenz: positive Immunreaktion in den Interzellularräumen der Epidermis für IgG (gesamte Epidermis) und C3 (basale $2 /{ }_{3}$ der Epidermis) sowie granulär entlang der epidermalen Basalmembran für C3.

Laborbefunde: $\mathrm{Hb}$ 6,9 mmol/l, Hk 0,34, Ery 4,02 Tpt/l, MCH 1,72 fmol, Leuko 17,29 Gpt/l, Thrombo 525,0 Gpt/l, Retikulozyten 1,8\%, Neutrophile 78,1\%, Lymphozyten 11,8\%, D-Dimer 0,880 mg/l, CRP 112,9 mg/l, LDH 4,85 $\mu \mathrm{kat} / \mathrm{l}$, Eisen 2,3 umol/l, TSH 0,127 mU/l, Elpho: Gammaglobuline 19,4\%, IgE 4310 U/ml, Pemphigus-AK $1: 1280$.

Therapie und Verlauf: Es wurde eine immunsuppressive Therapie mit Prednisolon $1 \mathrm{mg} / \mathrm{kg}$ KG und Tag begonnen. Nach 5-tägiger Therapie setzte eine Stabilisierung des Hautbefundes ein. Bei bestehender Dyspnoe/COPD sowie Infektanfälligkeit der Patientin und MRSA-Besiedlung der Haut waren wir mit dem Einsatz eines weiteren Immunsuppressivum zunächst zögerlich, gaben im Verlauf Azathioprin $150 \mathrm{mg} /$ die dazu, um Prednisolon bei Adipositas und zu befürchtendem kortikosteroidinduzierten Diabetes mellitus rascher reduzieren zu können. Zur Lokalbehandlung verwendeten wir Betamethason-V-Creme 0,1\% NRF, anfänglich in Kombination mit Lotio zinci oxydati. Zur Linderung des Juckreiz erhielt die Patientin in den ersten Tagen Levoceterizin (Xusal ${ }^{\circledR} 2 \times 1 \mathrm{Tbl}$./die). Unter dieser Behandlung heilten die Hautveränderungen stabil ab, bei Entlassung waren die Pemphigus-AK bereits um eine Titerstufe auf 1:640 abgefallen.

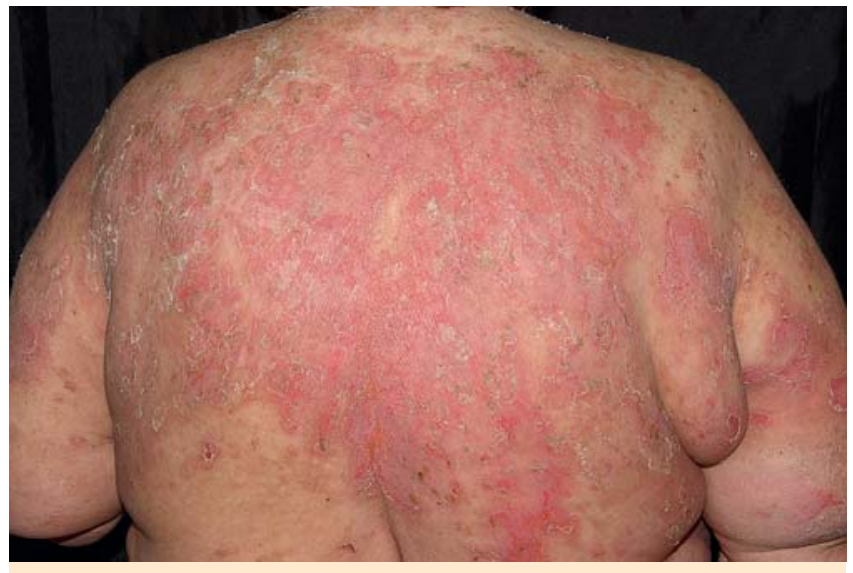

Abb. 7 Klinisches Bild des Pemphigus foliaceus.

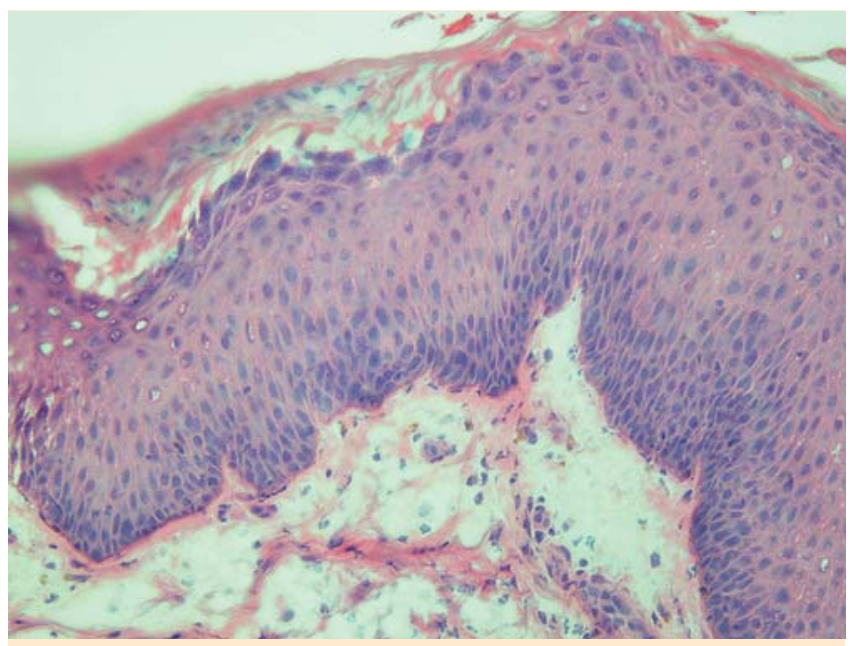

Abb. 8 Pemphigus foliaceus - Histologie $(\mathrm{HE}, \times 20)$.

Prednisolon konnte bis zu diesem Zeitpunkt auf $60 \mathrm{mg} / \mathrm{die}$ reduziert werden. Azathioprin wurde belassen.

Bei einem kurzen Kontakt im Januar 2009 bestätigte uns die Patientin einen guten Hautbefund. Azathioprin war ambulant wegen erhöhter Leberwerte reduziert worden, während vom Prednisolon noch $30 \mathrm{mg} / \mathrm{d}$ eingenommen werden.

Kommentar: Der Pemphigus foliaceus macht in Mitteleuropa 20\% der Pemphiguserkrankungen aus. Die Erkrankung beginnt am behaarten Kopf, im Gesicht und der vorderen sowie hinteren Schweißrinne. Wegen der subkutanen Spaltbildung und der damit verbundenen dünnen Blasendecke wird die Primäreffloreszenz „schlaffe Blase“ kaum gesehen. Im Verlauf entwickeln sich blätterteigartige Schuppenkrusten. Ausbreitung bis zur Erythrodermie ist möglich. Schleimhautbeteiligung fehlt. Die Läsionen besiedeln sekundär mit Bakterien, die durch Zersetzung des Wundsekretes einen unangenehmen Foetor erzeugen. In der Regel ist das Nikolski-Phänomen positiv, UV-Provozierbarkeit wird beschrieben. Serumantikörper binden bei Pemphigus foliaceus an das desmosomale Glykoprotein Desmoglein 1 [2]. Differenzialdiagnostisch müssen seborrhoisches Ekzem, subakut kutaner Lupus erythematodes und medikamenteninduzierter Pemphigus bedacht werden.

In der Behandlung stehen systemische Steroide in der Praxis an erster Stelle. In einer Meta-Analyse der Cochrane-Group ergab sich, dass Mycophenolat-Mofetil wirksamer ist als Azathioprin. 
Azathioprin und Cyclophosphamid haben steroidsparende Effekte gezeigt. Prospektive Studien wären aufgrund der mangelhaften Datenlage jedoch dringend erforderlich [1].

\section{Literatur \\ 1 Martin LK, Werth V, Villanueva E, Segall J, Murrell DF. Interventions for pemphigus vulagris and pemphigus foliaceus. Cochrane Database Syst Rev 2009; 1: CD006263 \\ 2 Sitaru C, Goebeler M, Zillikens D. Bullöse Autoimmundermatosen (I): Pathogenese und Diagnostik. J Dtsch Dermatol Ges 2004; 2: 123 - 139}

\section{Solitäre Mycosis fungoides}

$\nabla$

\section{I.-S.Schulze, J. Schönlebe, U. Wollina}

Es handelt sich um einen 43-jährigen Patienten, der in der Umgebung des Nabels einen Zeckenstich bemerkt hatte. Seit ca. vier Jahren bestehen in diesem Bereich Hautveränderungen.

Hautbefund: Es fanden sich periumbilikal bis zur rechten Abdominalhälfte ein handtellergroßes erythematöses Infiltrat mit Schuppen und teilweise follikulären Papeln sowie distal davon ein ca. 50-Centstück-großer schuppender Plaque ( Abb.9).

Histologie: (HE, Giemsa, PAS, Eisenreaktion, CD 3, CD 4, CD 5, CD 8, CD 20): In der retikulären Dermis ein vordergründig breites, bandförmiges, oberflächlich interstitiell sowie oberflächlich und tief perivaskulär, aber auch perifollikulär und perineural lokalisiertes Infiltrat aus fast ausschließlich T-Zell-reaktiven Lymphozyten (CD 3- und CD 5-positiv) mit deutlichem Überwiegen CD 4-positiver-T-Helferzellen (ca. 90\%) mit Beimengungen von wenigen CD 20-positiven Lymphozyten, monozytoiden/histiozytären Zellen, gelegentlich eosinophilen Granulozyten sowie Plasmazellen in wechselnder Zahl. Daneben hin und wieder diskrete Erythrozytenextravasate. Es besteht ein geringgradiger überwiegend einzelzelliger Infiltratepidermotropismus mit abschnittsweise perlschnurartig, innerhalb der Basalzellreihe nachweisbaren Lymphozyten (gelegentlich auch mit Halo) und einzelnen kleinen Lymphozytenclustern intraepidermal und einem Pautrierschen Mikroabszess. Ein Haarfollikel wird ebenfalls infiltriert. Keine nennenswerten Größenunterschiede zwischen intraepidermalen und dermalen Infiltratzellen.

Bildgebende Diagnostik: Sonografie von Abdomen, Axillen und Leisten ohne pathologischen Befund, insbesondere keine Lymphknotenveränderungen.

Rö-Thorax: Unauffälliger Befund, keine pathologischen Veränderungen.

Therapie und Verlauf: Unter der stationären Einleitung der Therapie mit Creme-PUVA und Betamethason-V-Creme 0,1\% NRF war nach einer Woche eine deutliche Befundbesserung zu verzeichnen, sodass die ambulane Fortsetzung der lokalen Fotochemotherapie empfohlen werden konnte. Nach ca. 50 Behandlungen fand sich nur ein Resterythem mit lichenoider Schuppung.

Kommentar: Wir stellen einen Patienten mit einem solitären Plaque einer Mycosis fungoides vor. Einige Autoren sind der Auffassung, dass die solitäre Mycosis fungoides eine eigene Entität verkörpert [3]. Unter den publizierten Fällen sind Patienten unter 18 Jahren häufiger zu finden. Sowohl Plaques als auch Noduli sind beschrieben worden $[2,3]$.

Da bei T-Zell-Lymphomen das klinische Bild in den meisten Fällen durch multiple Herde charakterisiert wird, stellt das Auftreten eines Solitärherdes eine Besonderheit dar. Unter den Patienten der eigenen Klinik waren in den letzten 6 Jahren insgesamt

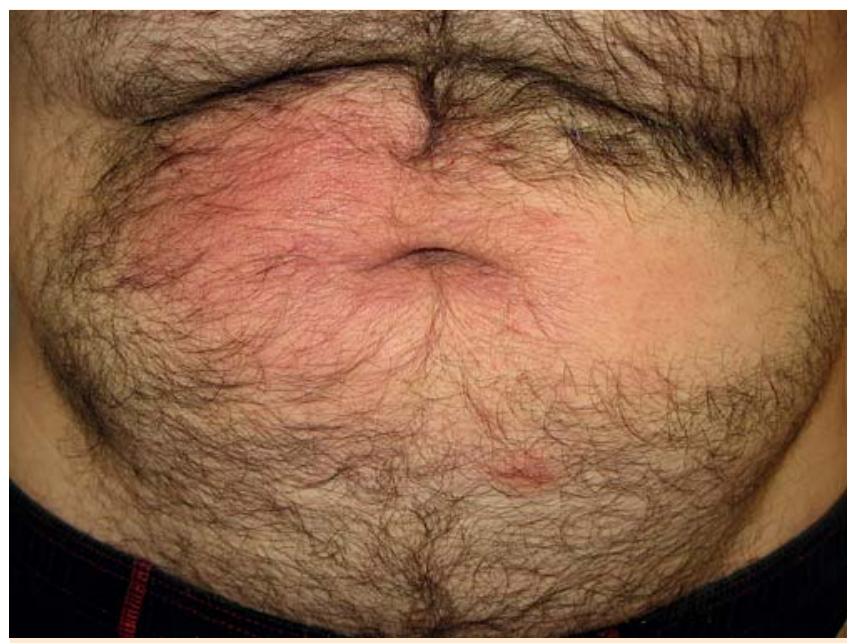

Abb. 9 Solitäre Mycosis fungoides.

nur 3 (einschließlich des aktuellen Falles), darunter 2 Männer und eine Frau.

Auf die wichtige Rolle der diagnostischen Exzision bei der Diagnosefindung soll hier hingewiesen werden, denn aufgrund der Anamnese stand die Differenzialdiagnose Erythema chronicum migrans zur Diskussion. Im Gegensatz zu B-Zell-Lymphomen wird bei T-Zell-Lymphomen ein Zusammenhang zu BorrelienInfektion nicht beschrieben.

Mit der Creme-PUVA steht eine geeignete, weil auch bei Langzeitanwendung nebenwirkungsarme Therapie, zur Verfügung [1].

\section{Literatur}

1 Hölzle E, Hönigsmann H, Röcken M, Ghoreschi K, Lehmann P. Empfehlungen zur Phototherapie und Photochemotherapie. J Dtsch Dermatol Ges 2003; 1: 985-997

2 Hodak E, Phenig E, Amichai B et al. Unilesional mycosis fungoides: a study of seven cases. Dermatology 2000; 201: 300-306

3 Oliver GF, Winkelmann RK. Unilesional mycosis fungoides: a distinct entity. J Am Acad Dermatol 1889; 20: 63-70

\section{Klassisches Kaposi-Sarkom \\ $\nabla$}

\section{A. Koch, J. Schönlebe, U. Wollina}

Im Februar 2008 bemerkte der ansonsten gesunde 51-jährige Patient erstmals kleine livid bläuliche Knoten im Bereich des rechten lateralen Malleolus. Es bestanden weder Juckreiz noch Schmerzen.

Hautbefund: Es fand sich am rechten lateralen Malleolus eine reizlose Narbe. Im ersten Interdigitalraum (IDR) der linken Hand konnte eine derbe Resistenz von 6 mm Größe aufgefunden werden. Farblich imponierte der Herd nur unwesentlich verändert gegenüber der normalen Umgebungshaut. Auffällig war jedoch ein deutliches Infiltrat. An der rechten medialen Fußkante zeigte sich ebenfalls noch eine leicht gerötete Effloreszenz, die sich klinisch nicht sicher einordnen ließ.

Histologie: Die histologische Aufarbeitung des Originalpräparates vom Malleolus lateralis erbrachte einen exulzerierten spindelzelligen Tumor mit erythrozytenhaltigen Lücken und Spaltbildungen sowie mehreren Kernteilungsfiguren ( $\triangle$ Abb.10a). 
Immunhistochemisch konnte bei den spindeligen Zellverbänden CD 34 nachgewiesen werden. Des Weiteren wurde eine Assoziation mit dem humanen Herpesvirus Typ 8 gefunden. Damit liegt ein Kaposi-Sarkom vor.

Das Exzisat von der Hand zeigte einen intradermal gelegenen knotig umschriebenen teilweise pseudokapselartig bindegewebig begrenzten Tumor aus irregulär angeordneten CD 31- und CD 34-positiven, teils mitotischen Spindelzellen, die teilweise mit Erythrozyten ausgefüllte blitzfigurenartige Spalträume einschließen ( Abb. 10b).

Laborbefunde: Pathologische Auffälligkeiten gab es bei den durchgeführten Untersuchungen (Blutbild, HIV-Serologie, Immunstatus) nicht.

Bildgebende Diagnostik: Unauffällig.

Therapie und Verlauf: Im April des Jahres suchte der Patient aufgrund des weiteren Progresses der Effloreszenz einen Chirurgen auf. Dieser entfernte den auf ca. $8 \mathrm{~mm}$ Durchmesser gewachsenen kutanen Tumor in Lokalanästhesie.

Die Entfernung der im weiteren Verlauf gefundenen Hautveränderungen erfolgte im Juni 2008 in unserer Klinik. Alle Tumoren konnten in sano exzidiert werden. Hinweise für ein erneutes Auftreten des Kaposi-Sarkoms nach über acht monatiger Nachbeobachtung gibt es derzeit nicht. Der Patient befindet sich in einer Dispensaire-Betreuung beim niedergelassenen Hautfacharzt, sodass Rezidive zeitnah erkannt und behandelt werden können.

Kommentar: Das Kaposi-Sarkom gilt als primär multilokuläre Systemerkrankung und gehört zu den malignen Gefäßtumoren. Mit einer Inzidenz von 1 Fall pro 10 Mio. Einwohnern und Jahr ist es ein sehr seltener Tumor [4].

Das hier demonstrierte klassische Kaposi-Sarkom gilt als gering maligner und langsam progredienter Tumor. Die Prognose ist günstig. Bezüglich der Lokalisation zeigt sich eine Bevorzugung der unteren Extremitäten [3].

Hinsichtlich der Ätiologie ist bislang bekannt, dass bei allen Formen eine Infektion mit dem HHV-8-Virus vorliegen soll. Man unterscheidet vier Formen:

1. chronisches oder klassisches Kaposi-Sarkom (gehäuft in Osteuropa, Italien, Äquatorialafrika)

2. Kaposi-Sarkom bei Immunsuppression (Organtransplantation)

3. endemisches Kaposi-Sarkom (Afrika)

4. epidemisches Kaposi-Sarkom (HIV-assoziiert) [1,2]

Das Kaposi-Sarkom ist, wie bei unserem Patienten, klinisch durch initial auftretende asymptomatische lividrote Makulae oder Knoten gekennzeichnet, die sich in den Hautspaltlinien anordnen können. Konfluierende Plaques und infiltrativ wachsene Knoten sind oft von Ödemen begleitet.

Bezüglich histologischer Merkmale sind das klassische und das Kaposi-Sarkom bei Immundefizienz durch einzelne Besonderheiten charakterisiert. Beim klassischen Typ ist eine pseudokapsuläre Ummantelung der Tumorknoten typisch, während der immundefiziente Typ frühzeitig durch ein inflitratives Wachstum gekennzeichnet ist, durch Kolonisation der periadnexiellen und perineuronalen Dermis und intravaskuläre papilläre Projektionen atypischer Endothelien [1,2]. Immunhistochemisch ist es möglich, HHV-8-LNA (latent nuclear antigen) im formalinfixierten und paraffineingebetteten Material einfach und zuverlässig nachzuweisen.

Für die Behandlung des Kaposi-Sarkoms gibt es keine allgemein anerkannten „Standardtherapien“. Bei isolierten kutanen Herden bietet sich die Operation mit entsprechendem Sicherheits-

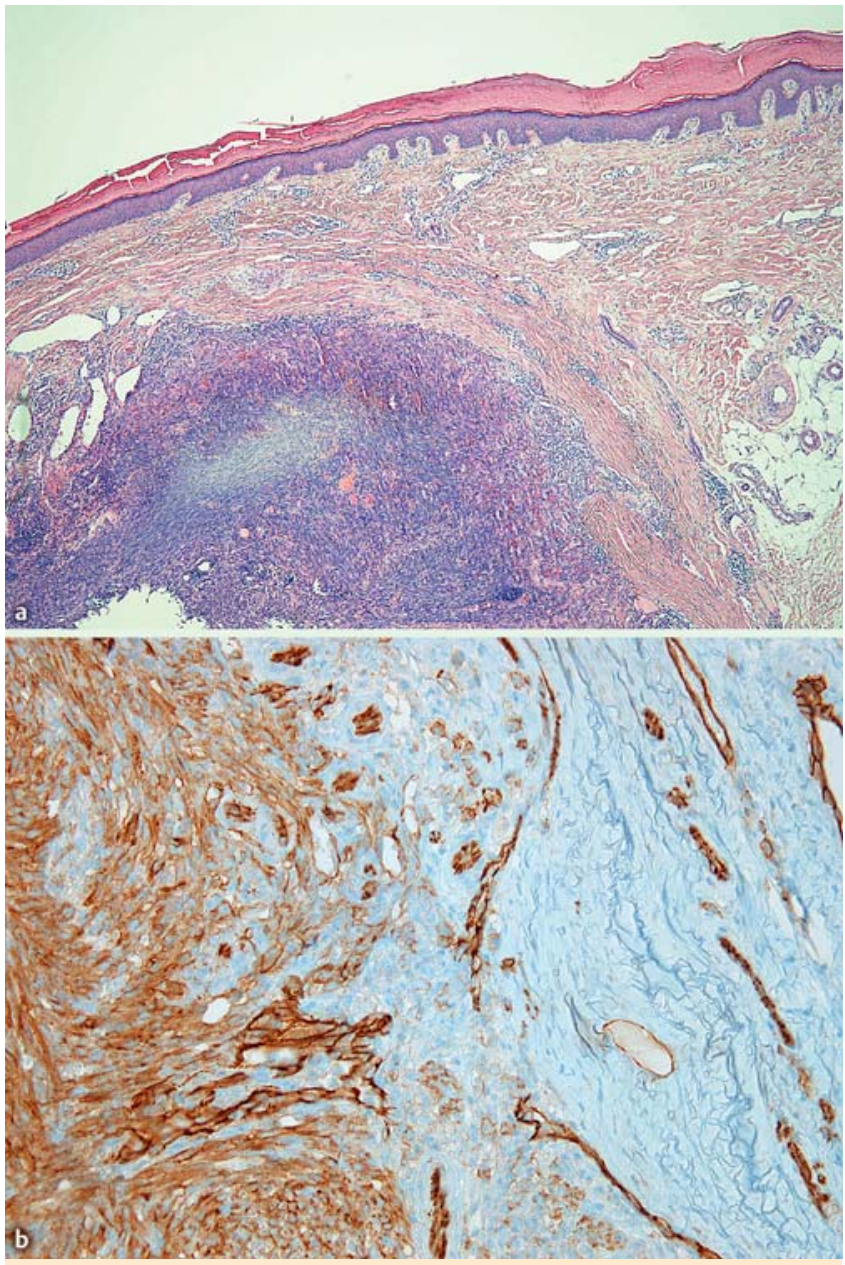

Abb. 10 Klassisches Kaposi-Sarkom. (a) Übersicht mit dermalem Knoten umgeben von einer Pseudokapsel (HE, × 4); (b) Detail, Immunhistologie (CD 34, × 20).

abstand an. Ansonsten haben sich die Radiatio (Röntgenweichstrahltherapie, schnelle Elektronen, Kobaltbestrahlung) sowie lokale Chemo- und Immuntherapien (Interferon alpha intraläsional, Retinoide, Vincaalkaloide, Imiquimod) bewährt. Bei fortgeschrittenem Tumorstadium wird erfolgreich liposomales Doxorubicin eingesetzt $[3,5]$. Aufgrund der Seltenheit des KaposiSarkoms liegen keine großen Erfahrungen hinsichtlich einer suffizienten Nachsorge vor. Es wird jedoch empfohlen, je nach Erkrankungsstadium, klinische Kontrollen in drei bis sechs monatigen Abständen einzuhalten [5].

\section{Literatur}

1 Castelli E, Wollina U. Mediterranean and immunodeficiency associated Kaposi's sarcoma - does micromorpholgy reflect clinical patterns? A clinicopathologic study with phenotypic characterization of tumor cells. Oncol Rep 1997; 4: 289-295

2 Castelli E, Wollina $U$. Histopathologic features of progression in mediterranean and immunodeficiency-related Kaposi sarcoma. Am J Dermatopathol 2000; 22: 89-91

3 Di Lorenzo G. Update on classic Kaposi sarcoma therapy: new look at an old disease. Crit Rev Oncol Hematol 2008; 68: 242 - 249

4 Pantanowitz L, Mullen J, Dezube BJ. Primary Kaposi sarcoma of the subcutaneous tissue. World J Surg Oncol 2008; 6: 94

5 Vogt T, Brockmeyer N, Kutzner H, Schöfer H. Short German guidelines: Angiosarcoma and Kaposi sarcoma. J Dtsch Dermatol Ges 2008; 6 (Suppl 1): 19-24 


\section{Prätibiale Panniculitis ossificans}

\section{$\nabla$}

\section{A. Koch, J. Schönlebe}

Im Juni des Jahres 2008 bemerkte die 30-jährige Patientin eine schmerzlose derbe Resistenz am linken Unterschenkel. Der Tumor zeigte im weiteren Verlauf eine deutliche Wachstumstendenz. Eine Verletzung oder ein anderes Trauma war der Patientin nicht erinnerlich. Zur Abklärung der atypischen Resistenz erfolgten im November 2008 mehrere tiefe Biopsien, die differenzialdiagnostisch den Verdacht auf ein mögliches malignes Chondrosarkom erbrachten. Somit war eine vollständige Entfernung der tumorösen Veränderung unumgänglich.

Hautbefund: Am linken proximalen Unterschenkel prätibial fiel eine schmerzlose derbe subkutan gelegene multinodöse Resistenz auf, die als gut verschieblich imponierte. Sie zeigte mit $5 \times 4 \mathrm{~cm}$ Größe in der Flächenausdehnung und knapp $2 \mathrm{~cm}$ Erhabenheit beeindruckende Abmessungen. Die Haut über dem Tumor war leicht mit dem Untergrund verbacken.

\section{Histologie:}

Biopsie: Verdächtig auf einen ossifizierenden fibromyxoiden Tumor des Weichgewebes. Differenzialdiagnostisch muss noch an ein myxoides Chondrosarkom gedacht werden, was anhand der vorliegenden Biopsie jedoch nicht zu beweisen ist.

Exzisat: Es zeigt sich eine verkalkte Fettgewebsnekrose mit teilweise myxoid aufgelockertem relativ zell- und kapillarreichem Gewebe mit dominierender Spindelzellkomponente. Zelluläre Atypien oder mitotische Aktivität sind nicht nachweisbar. Weiterhin lassen sich Kollagenfaser- und Osteoidbildungen nachweisen, die eine deutliche Reifung von Knochengewebe mit Ausbildung von reifem Lamellenknochen aufzeigen. Die Faszie und die Skelettmuskulatur werden von der Läsion nicht mit erfasst.

\section{Bildgebende Diagnostik:}

Röntgen linker Unterschenkel: $13 \mathrm{~mm}$ große Verkalkung ventral der Tibia.

MRT linker Unterschenkel: Inhomogene, intensiv Kontrastmittel aufnehmende Raumforderung im subkutanen Fettgewebe ventral der Tibia von $3 \times 2 \mathrm{~cm}$ und einer sagittalen Dicke von 6-7 mm. Kein Anhalt für eine Knochen- oder Muskelinfiltration. Therapie und Verlauf: Nach bioptischer Sicherung und apparativer Diagnostik wurde die Indikation zur radikalen Resektion gestellt. Im Januar 2009 führten die chirurgischen Kollegen den Einriff durch. Der Defekt wurde plastisch verschlossen. Nach histologischer Aufarbeitung des gesamten Präparates konnte der Malignitätsverdacht ausgeräumt werden. Letztendlich handelte es sich um eine ossifizierende Pannikulitis.

Kommentar: Die Panniculitis ossificans ist eine relativ selten auftretende Erkrankung des subkutanen Fettgewebes und wird eigentlich durch ein stattgehabtes Trauma ausgelöst [1]. In unserem Fall war jedoch keine Verletzung erinnerlich, sodass der Auslöser unklar bleibt. Die meisten Beschreibungen von ossifizierender Pannikulitis betreffen die unteren Extremitäten. Die Erkrankung wird als Teil des Spektrums der kutanen Kalziphylxie eingeordnet, welche auch bei Fehlen renaler Erkrankungen auftreten kann [2,3]. Histologisch ist die kutane Kalziphylaxie durch eine kalzifizierende septale Pannikulitis sowohl in frühen als auch spätreren Stadien charakterisiert [3].

Als Therapie der Wahl steht die operative Sanierung an erster Stelle [1]. Differenzialdiagnostisch muss bei relativ rasch wachsenden Tumoren immer ein maligner Prozess ausgeschlossen werden. Aufgrund der Derbheit und der palpablen Multinodalität war hier auch an ein Dermatofibrosarcoma protuberans zu denken.

\section{Literatur}

1 Burke GA, Shah D, MacBean AD. Panniculitis ossificans traumatica: an unusual presentation. Br J Oral Maxillofac Surg 2008;46: 596-598

2 Campanelli A, Kaya G, Masouyé I, Borradori L. Calcifying panniculitis following subcutaneous injections of nadroparin-calcium in a patient with osteomalacia. Br J Dermatol 2005;153: 657-660

3 Essary LR, Wick MR. Cutaneous calciphylaxis. An underrecognized clinicopathologic entity. Am J Clin Pathol 2000;113: 280-287

\section{Paraneoplastische Dermatomyositis}

\section{A. Gemmeke, T. Kittner}

Zur Aufnahme gelangte ein 74-jähriger Patient, der seit 4 Wochen über eine zunehmende Ermüdbarkeit und proximal betonte Muskelschwäche klagte. Anamnestisch waren ein Diabetes mellitus-Typ II, eine arterielle Hypertonie und eine Hyperurikämie bekannt.

Hautbefund: Flächige Erytheme am Rücken, unscharf begrenzt. Im Bereich des Dekolletes teils krustös belegte, teils nässende Erytheme ( Abb. 11). Kein heliotropes Liderythem, keine Gottronschen Papeln.

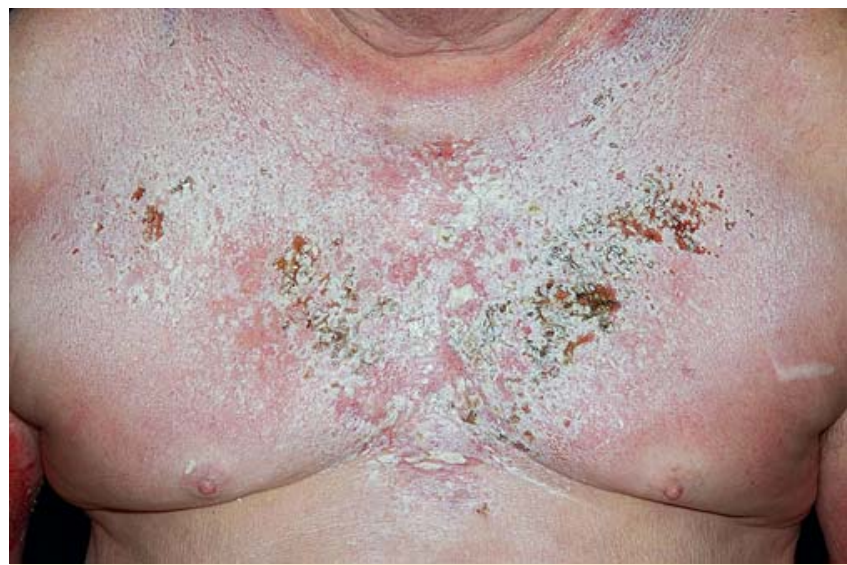

Abb. 11 Paraneoplastische Dermatomyositis.

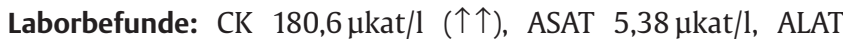
$1,33 \mu \mathrm{kat} / \mathrm{l}$, LDH $11,16 \mu \mathrm{kat} / \mathrm{l}$, Neuronen-spezifische Enolase (NSE) $69 \mu \mathrm{g} / \mathrm{l}(\uparrow)$.

Weiterführende Untersuchungen: ENG/EMG: M. deltoideus Summenpotenzial mit niedriger Amplitude.

Gastroskopie, Koloskopie, CT Abdomen, HNO: unauffällig.

CT Thorax: Bronchialkarzinom im linken Unterlappen mit V.a. Hilus-Lymphknotenmetastasen und V.a. pulmonale Metastase rechts.

Therapie und Verlauf: Unter der Diagnose einer paraneoplastischen Dermatomyositis erfolgte die Prednisolon-Stoßtherapie mit 100 mg/d i.v. in Kombination mit einer topischen Kortisonbehandlung (Jellin-Neomycin-Salbe ${ }^{\circledR}$ ).

Nach Feststellung des Tumors wurde im Tumorboard die operative bzw. chemotherapeutische Behandlung besprochen. Jedoch musste der Patient notfallmäßig in die Medizinische Klinik verlegt werden, da sich eine Sepsis durch Pseudomonas aeruginosa entwickelt hatte. Der Patient verstarb an den Folgen eines septischen Schocks. Eine Obduktion wurde von den Angehörigen abgelehnt. 
Diskussion: Die Dermatomyositis kann bei den über 35-Jährigen als Paraneoplasie auftreten. Dabei wird sie überwiegend vor dem Tumorleiden symptomatisch. Eine Tumorsuche ist somit angezeigt.

Von den klassischen Diagnosekriterien der Dermatomyositis bot unser Patient die proximal betonte Muskelschwäche, eine Erhöhung der Muskelenzyme sowie einen Teil der charakteristischen Hautveränderungen („Schultertuch“) [1]. Im Rahmen der Umgebungsuntersuchungen wurde ein Lungenkrebs entdeckt. Der Lungenkrebs gehört zu den häufigen mit der Dermatomyositis assoziierten Tumoren. Darüberhinaus sind Tumoren des Gastrointestinaltraktes, der Ovarien und das Non-Hodgkin-Lymphom bei der Dermatomyositis beobachtet worden [2-4].

\section{Literatur}

1 Bohan A, Peter JB. Polymyositis and dermatomyositis. Patrs 1 and 2. N Engl J Med 1975; 292: 344 - 347, 403 - 407

2 Gabrilovich M, Raza M, Dolan S, Raza T. Paraneoplastic polymyositis associated with squamous cell carcinoma of the lung. Chest 2006; 129: $1721-1723$

3 Przybylski G, Jarzemska A, Czerniak J et al. A case of a patient with dermatomyositis as a prodromal sign of lung cancer. Pol Arch Med Wewn 2008; $118: 143-147$

4 Wilmer A, Lange D, Mentzel T, Wollina U. Paraneoplastische Dermatomyositis bei Magenkarzinom. Akt Chirurgie 1996; 31: 51-54

\section{Porokeratosis Mibelli gigantea nach Warzentherapie}

M. Tilp, J. Schönlebe

Seit Oktober 2006 leidet die 70-jährige Patientin unter stark juckenden verrukösen Hautveränderungen am linken Unterschenkel, welche sich flächig auf beide Unterschenkel ausbreiteten. Histologisch (3/2007) stellten sich damals Verrucae vulgaris dar, welche mittels Laser und Kürettage entfernt wurden. Im Verlauf kam es zu nunmehr schmerzhaften Rezidiven an beiden Unterschenkeln. Eine erneute ambulante diagnostische Exzision ergab im Juni 2008 den Befund einer Porokeratosis Mibelli mit HPV-Induktion. Innerhalb von 4 Wochen traten die Hautveränderungen zusätzlich an beiden Händen und Armen auf. Die Patientin klagte über eine ausgesprochene schmerzhafte Berührungsempfindlichkeit.

Als Nebendiagnosen waren bekannt: Polymyalgia rheumatica, chronische Niereninsuffizienz im Stadium der kompensierten Retention, metabolisches Syndrom und Osteoporose.

Hautbefund: Es fanden sich multiple kreisrunde, unterschiedlich große erythematöse Plaques mit ausgeprägtem hyperkeratotischem Randwall an beiden Unterschenkeln und Füßen. An den Unterarmen fielen beginnende, stecknadelkopfgroße, runde Herde mit dunkelbrauner Umrandung auf ( $\bullet$ Abb. 12).

Histologie: Verrukiforme Hautläsion mit teils kirchturmspitzenartiger Papillomatose sowie einzelnen säulenförmigen Parakeratosen.

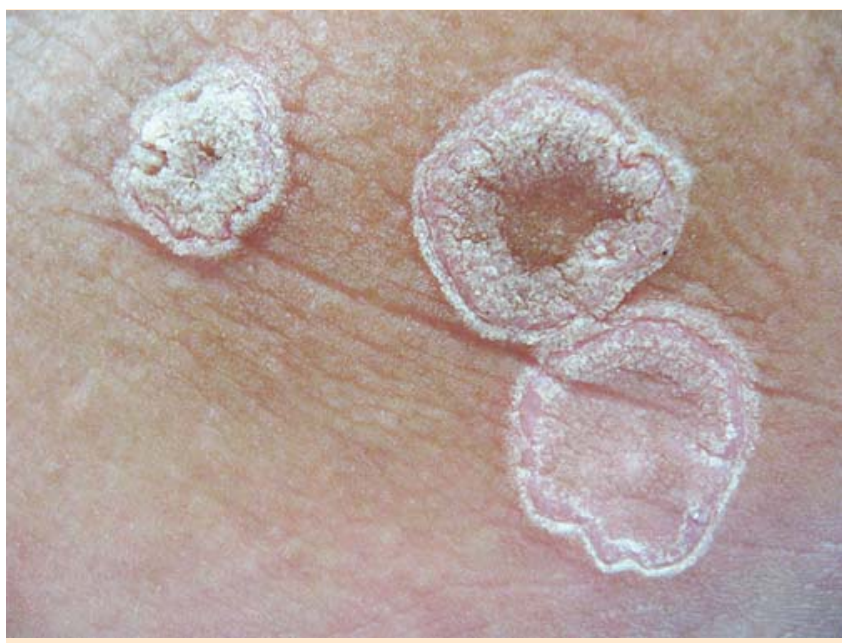

Abb. 12 Porokeratosis Mibelli gigantea.

Therapie und Verlauf: Die Lokaltherapie mit Retinoiden war nicht wirksam. Deshalb wurde die Patientin auf Acitretin (Neotigason $^{\circledR} 10 \mathrm{mg} / \mathrm{d}$ ) eingestellt. Wegen eines Erysipels Ende August 2008 wurde die Acitretintherapie unterbrochen. Danach kam es zur massiven Verschlechterung des Hautbefundes und zur Entwicklung neuer Läsionen. Wir setzten die Behandlung erneut an und erhöhten wegen mangelhaften Ansprechens die Dosierung auf $20 \mathrm{mg} / \mathrm{d}$.

Kommentar: Bei der nach Mibelli benannten Porokeratose handelt es sich um eine multifokal auftretende, umschriebene Differenzierungsstörung der Epidermis mit Parakeratose.

Die Vererbung ist autosomal-dominant, sporadisches Auftreten kommt vor. Männer sind bevorzugt betroffen $(2: 1)$. Prädilektionsstellen sind die Extremitäten, Gesicht, Genitalien, Mundschleimhaut und die Kornea. Klinisch imponieren inital kleine Papeln mit zentralem Hornstachel. Diese nehmen im Verlauf an Größe zu und bilden schließlich eine Fläche normaler bis leicht atrophischer Haut, welche von einer weißlichen Kerbe umrahmt ist, in welche zaunartig eine Hornlamelle eingesenkt ist. Die Herde sind rundlich zirzinär oder girlandenartig umrandet. Histologisch typisch sind die schlotförmige Parakeratose und die kornoide Lamelle [2]. Die Porokeratosis Mibelli gigantea ist eine morphologische Variante der Porokeratose [1]. Die Erkrankung weist eine langsame Progredienz auf, spontane Regression mit leicht atrophen Narben kommt vor. Eine Karzinomentwicklung mit Auftreten von Morbus Bowen, Basalzellkarzinom oder Plattenepithelkarzinom ist möglich.

Die alleinige lokale Therapie ist meist frustran.

\section{Literatur}

1 Götz A, Kopera D, Wach F, Hohenleutner U, Landthaler M. Porokeratosis Mibelli gigantea. Hautarzt 1999; 50: 435-438

2 Pizzichetta MA, Canzonieri V, Massone C, Soyer HP. Clinical and dermoscopic features of porokeratosis of Mibelli. Arch Dermatol 2009; 145: $91-92$ 